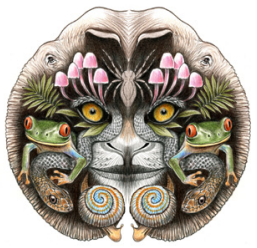

ISSN 0974-7907 (Online) ISSN 0974-7893 (Print)

OPEN ACCESS

\title{
PREVALENCE OF INTESTINAL PARASITIC INFECTIONS IN FREE-RANGING Red PANdA AIluRus fulgens CuVIER, 1825 (MAMmAliA: CARNIVORA: AILURIDAE) IN NePAL
}

\section{Sonam Tashi Lama ${ }^{1}$, Rinzin Phunjok Lama ${ }^{2}$, Ganga Ram Regmi ${ }^{3}$ \& Tirth Raj Ghimire ${ }^{4}$}

1,2,3,4 Global Primate Network, GPO Box: 26288, Kathmandu, Nepal ${ }^{1}$ Red Panda Network, Baluwatar, Kathmandu, Nepa ${ }^{4}$ Department of Zoology, Birendra Multiple Campus, Tribhuvan University, Chitwan, Nepal 1sonam.tac@gmail.com, ${ }^{2}$ phunjok@hotmail.com, ${ }^{3}$ regmigr1978@gmail.com, ${ }^{4}$ tirthprimate@gmail.com (corresponding author)

Abstract: The Red Panda Ailurus fulgens is a small carnivore that is adapted to a mainly herbivorous diet. The present study was conducted to investigate the prevalence of parasitic infections in a free-ranging population of Red Pandas in a community forest in Nepal. A total of 23 faecal samples were collected and examined. Protozoa infections were the most common and cestode infections occurred the least. Our findings suggest that parasites might be a significant problem for the health of the Red Pandas in the study area. Molecular methods should be used to further investigate the taxonomic position of the parasites and their role in threatening the resilience of Red Panda populations in Nepal.

Keywords: Angiostrongylus, Baylisascaris, conservation, Cyclospora, molluscs, Red Panda, Trichuris.
The Red Panda Ailurus fulgens Cuvier, 1825 is a shy and charismatic mammal occurring in Asia. It is an exceptional member of the order Carnivora as it is nearly entirely herbivorous, living largely on a diet of leaves and shoots of bamboo, berries, mushrooms (Yonzon \& Hunter 1991; Pradhan et al. 2001) and occasionally small mammals, birds, eggs, insects, blossoms and acorns (Johnson et al. 1988). The IUCN Red List of Threatened Species classifies the Red Panda currently as 'Vulnerable' (Wang et al. 2008). This animal is found
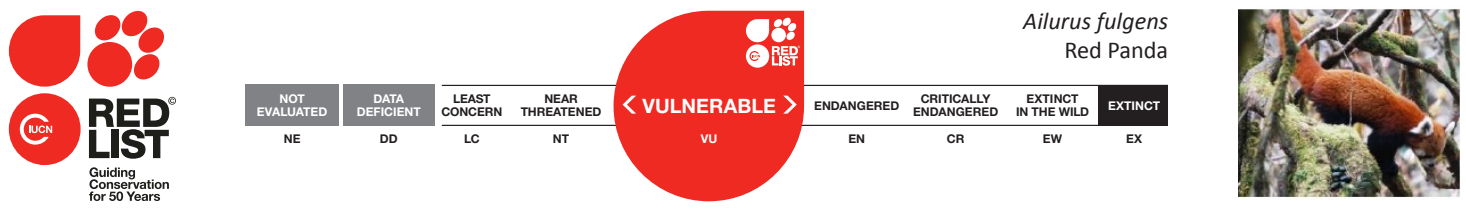

DOI: http://dx.doi.org/10.11609/JoTT.04208.7460-4

Editor: Ulrike Streicher, Wildlife Veterinarian / Wildlife Management Consultant, Eugene, USA.

Date of publication: 26 June 2015 (online \& print)

Manuscript details: Ms \# 04208 | Received 27 November 2014 | Final received 18 May 2015 | Finally accepted 05 June 2015

Citation: Lama, S.T., R.P. Lama, G.R. Regmi \& T.R. Ghimire (2015). Prevalence of intestinal parasitic infections in free-ranging Red Panda Ailurus fulgens Cuvier, 1825 (Mammalia: Carnivora: Ailuridae) in Nepal. Journal of Threatened Taxa 7(8): 7460-7464; http://dx.doi.org/10.11609/JoTT.04208.7460-4

Copyright: (c) Lama et al. 2015. Creative Commons Attribution 4.0 International License. JoTT allows unrestricted use of this article in any medium, reproduction and distribution by providing adequate credit to the authors and the source of publication.

Funding: Red Panda Network (Funding obtained in March 2013).

Competing interests: The authors declare no competing interests.

Acknowledgements: We acknowledge Dr. Mahesh Gajurel, Advanced Community Hospital, Chitwan, Nepal for providing laboratory facilities. We sincerely thank Mr. Suman Bhattarai, Institute of Forestry, Pokhara, Dr. Jhamak Bahadur Karki, Nepal Engineering College-Center for PostGraduate Studies, Pokhara University, and Rajeev Poudel and Damber Bista, Red Panda Network, Kathmandu for their support and guidance for the preliminary assessment of Red Panda, Mr. Gyajo Lama, Roshan KC and Robert Mahara for their assistance in surveying the Red Panda and in collecting faeces in the field, and Jonathan Hanson, PhD fellow at University of Cambridge for reviewing language of the manuscript. District Forest Office, Rolpa, provided the necessary research permit (751-2069-070 in 06/03/2013) for collecting the Red Panda faeces from the study area and provided necessary support.

RED PANDA NETWOR K
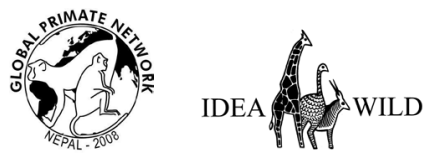
in the subtropical and temperate forests of Nepal, India, Bhutan, northern Myanmar and southwestern China (Choudhury 2001).

In recent years, infectious agents such as parasites transmitted by close contacts between wild and domestic animals may have contributed to the current extinction crisis, which wildlife faces globally (Smith et al. 2006; Pedersen et al. 2007). This may also be the case in the Red Panda as they have been reported to carry high numbers of parasites like Eimeria, Isospora, Toxoplasma gondii, Angiostrongylus spp., Crenosoma spp. and unknown metastrongyloid nematodes (Grondahl et al. 2005; Patterson-Kane et al. 2009) suggesting a possible link between these endoparasites and the morbidity and mortality of the pandas. A total of five Red Panda individuals were found dead due to unknown reasons in two community forests of the Taplejung District of eastern Nepal from February 2011 to September 2012. The significance of this issue was further emphasized by reports from local people who witnessed the deaths of a number of Red Pandas individuals in the forests adjacent to the study area. Thus, in addition to habitat destruction, degradation and fragmentation, poaching and wildlife trade (Yonzon \& Hunter 1991; Wei et al. 1999; Choudhury 2001; Pradhan et al. 2001; Steffens 2004; Williams 2006), parasitic infections may be a threat to the surviving Red Panda populations in the wild. However, very little information is available to date regarding parasitic infection in these animals, not only in Nepal but throughout their entire distribution range. Understanding the threats to Red Pandas and to develop effective conservation measures is a challenge for conservationists (Gonzalez-Suarez \& Revilla 2014). Thus, we conducted this study to investigate the prevalence of gastrointestinal (GI) parasites and to assess their possible effect on the health of Red Pandas.

\section{Materials AND MethodS}

\section{Study area and sample collection}

The study was conducted in the Kothi Bhir community forest area (altitudinal range: 2800-3200 m) in Gaam Village Development Committee, Rolpa District, situated in the mid-western region of Nepal. The presence of Red Pandas in this area was first confirmed by the first author (S.T. Lama) in collaboration with Red Panda Network in March 2013. A detailed ecological survey was conducted from 1 to 20 October 2013.

The presence of Red Panda was confirmed by direct observation and by its faeces. The number of faeces piles in a single defecation site is usually $8-15$, but $15-30$ or sometimes even more than 100 faeces piles are found in the repeatedly used sites, which are called latrines (Yonzon 1989). The line intercept method was used to collect the faeces of these animals (Sutherland 1996). Thus, a total of five line intercepts were established along altitudinal gradients at $100 \mathrm{~m}$ intervals in the study area. Altimeter and Global Positioning System (GPS) were used to record the altitude and position of the Red Panda faeces. Then, a total of 23 faecal samples were collected from the plots. The samples were collected with the help of spatulas, stored in vials and preserved in $2.5 \%$ potassium dichromate solution. Samples were stored at $4^{\circ} \mathrm{C}$ until analysis. The physical characteristics of the faeces (e.g., colour and consistency) and the defecation sites (e.g., ground, log or tree) were recorded.

\section{Sample examination}

The examinations were conducted in the laboratory of the Advanced Community Hospital, Chitwan, Nepal. Faecal samples were examined by direct light microscopy (100x and 400x) and a faecal smear was stained with modified acid-fast stain for examination of coccidia. The ocular stage micrometer was used to measure the size of the various coccidia such as Cyclospora (8-10 $\mathrm{\mu m}$ in diameter), Cryptosporidium (4-6 $\mu \mathrm{m}$ in diameter), Eimeria (10-40 $\mu \mathrm{m} \times 10-30 \mu \mathrm{m})$ and Isospora (20-33 $\mu \mathrm{m} \times 10-19 \mu \mathrm{m}$ in size) (Ghimire $\&$ Sherchan 2006). Sporulation assays were performed to confirm the presence of coccidian parasites. For this, samples positive for coccidia were stored at ambient temperature (approximately $23^{\circ} \mathrm{C}$ ) and were examined at regular intervals over a period of two weeks starting from the time of collection (Eberhard et al. 1997; Ghimire \& Sherchan 2006).

\section{RESULTS}

All 23 samples were found to be positive for gastrointestinal parasites (100\%) (Fig. 1). The occurrence rates for different groups of parasites were as follows: protozoa $100.0 \%$, nematodes $52.2 \%$, trematodes $13.0 \%$, and cestodes $4.3 \%$. Out of the 23 samples positive for protozoa, 16 samples contained protozoan species of a size ranging from 10-14 $\mu \mathrm{m}$ diameter, which we were unable to identify. Five other samples contained coccidian parasites of various oocyst characters. One sample contained oocysts from 4-5 $\mu \mathrm{m}$ in diameter resembling Cryptosporidium, one sample contained oocyst from 8-9 $\mu \mathrm{m}$ in diameter resembling Cyclospora. Out of 12 samples positive for nematodes, Trichuris spp. were found in six samples and Baylisascaris spp. were found in three samples. Larval forms resembling the first stage of Angiostrongylus larvae were found 


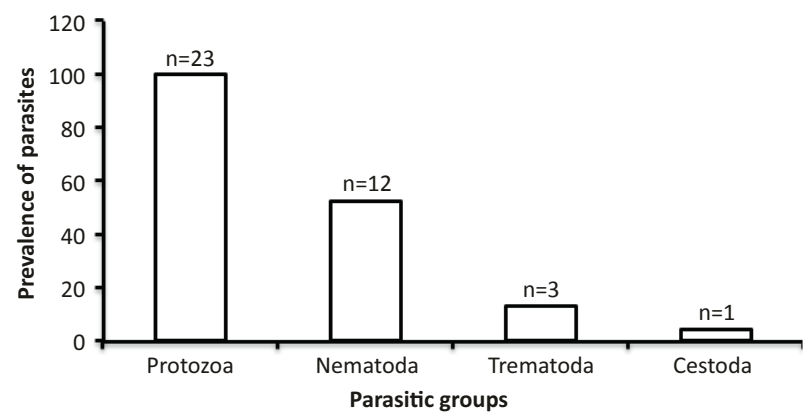

Figure 1. Prevalence of various GI parasitic groups in 23 stool samples collected from Red Panda in Gaam VDC, Rolpa, Nepal from October 1 to 20 October 2013. In each vertical bar, denominator (n = various) shows the total number of positive sample for specific parasitic group and numerator $(\mathbf{N}=23)$ represents the total number of stool samples. in two samples. They were identified based on their morphology as their slightly plump and filiform shape, their distinct anterior cephalic button, the broad based tail with a distinct dorsally placed notch and the spirally coiled distal end (Bertelsen et al. 2010). Unidentified eggs resembling nematode eggs were found in one sample. Three samples contained operculated eggs resembling those of trematodes (Fig. 2) and one sample contained eggs resembling those of cestodes.

\section{Discussion}

Wild animals serve as definitive and reservoir hosts of a wide range of parasites. This is illustrated by this study which found various GI parasites in all faecal samples of Red Pandas. Different factors may contribute to the high rate of GI parasites which can be discussed on the basis

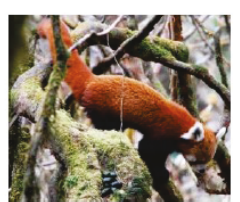

Pellet on tree

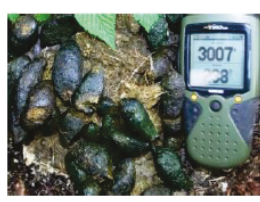

Pellet on ground

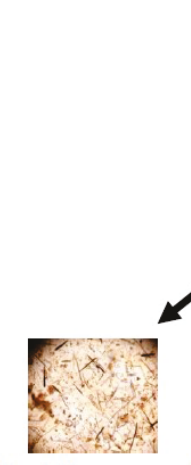

Plant tissues and tendrills (X400)

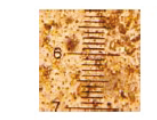

Cyclopora spp. (X400)

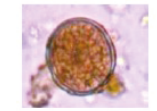

Coccidia (X400)

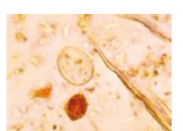

Egg of trematode (X400)

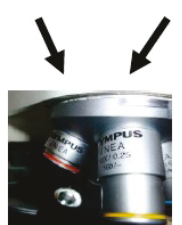

Microscopic examination
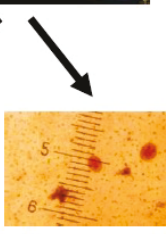

Unknown protozoa (X400)

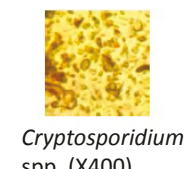
spp. (X400)

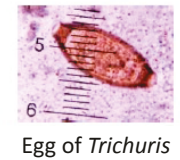
spp. (X400)

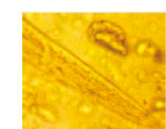

Larva of Angiotrongylus spp. (X400)
Six hypothetical routes of parasite

transmission in Red Panda
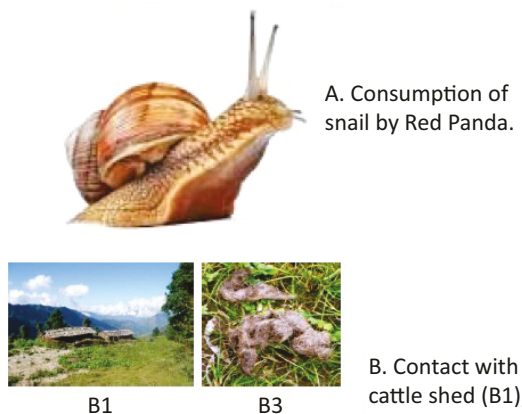

B. Contact with cattle in cattle shed (B1), cattle dung (B2), cat feces (B3), deer as shown by their

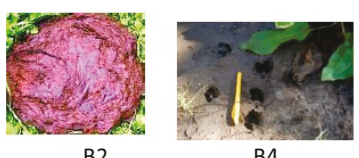

B2

B4
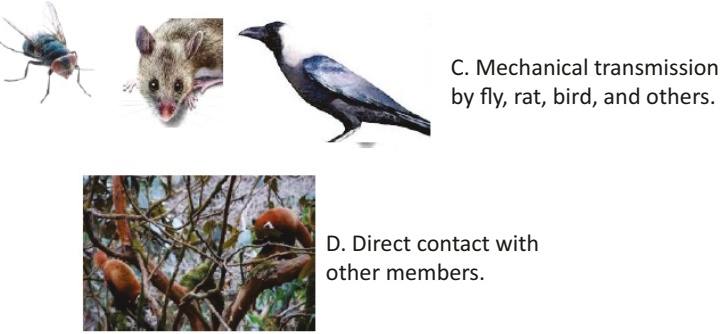

Direct contact with other members.

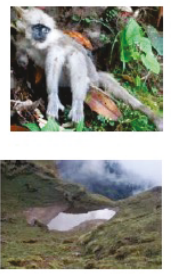

E. Contact with stool of primate (This is dead primate in the study area).

Contact with water body and surface rain.
Figure 2. Graphical summary showing the faeces collection, examination and detection of various parasites and summarizing the six hypothetical routes of parasite transmission in Red Panda in the current study area. 
of Red Panda behaviour and habitat.

The Red Panda defecates openly on the ground and on trees. Parasites can be easily transmitted via mechanical vectors (Fig. 2) like flies, rats, birds, lizards, cockroaches and beetles from one faecal matter to another. The Red Panda itself can be infected transcutaneously by larval forms when in search of food and water on ground contaminated with faeces. Pandas can also be infected through the consumption of vegetation and water contaminated with parasitic stages such as cysts, oocysts, eggs and larval forms. Infections can also occur while ingesting birds, insect and molluscs infected with trematodes or nematodes. We observed Red Pandas intentionally or accidentally ingesting snails along with bamboo leaves. Pandas need to drink water frequently and the areas around water are favourable habitat for these snails. Many trematodes and nematodes complete their asexual lifecycle in snails and slugs as the intermediate hosts. When the snails are ingested by the primary hosts, in this case the Red Panda, the larvae enter the digestive tract where they establish and mature. Both trematode eggs and molluscan shells were detected in the faecal sample and an epidemiological connection may exist between consumption of snails and parasitic infection in Red Panda. But consumption of whole snails may not be the sole cause of parasite transmission. For example, A. costaricensis was also found in patients after the consumption of food contaminated solely with mucous from infected molluscs (Zanini \& Graeff-Teixeira 1995; Kramer et al. 1998; Patterson-Kane et al. 2009).

During the mating season, the Red Panda lives in pairs (Nowak 1999), however it shows solitary behaviour for the rest of the time. That is why males usually have large territories that overlap with those of numerous females. This behaviour can assist in the dispersal of parasites in a wide range of occupied territories.

Licking or nuzzling the urine marks and faeces of conspecifics is probably also a common way of parasite transmission among these carnivores (Zhang et al. 2008).

Cattle, monkeys, dogs and domestic cats commonly visit the research area (Fig. 2) where cross-contamination via surface rain water or faecal matter can occur. This may be the reason why coccidia resembling the Cryptosporidium of cattle and the Cyclospora of primates as well as canid or felid Trichuris were found in the samples.

Baylisascaris, Trichuris and Angiostrongylus found in this study are parasites the pandas have in common with other carnivores in the forest ecosystem. Baylisascaris spp., a parasite favouring humans, utilizes more than
100 species of birds and mammals as hosts. These nematodes are pathologically very important because they appear as ocular, visceral and neural larvae migrans, resulting in blindness, loss of muscle control, hepatomegaly and coma. This parasite has been proven to be the underlying cause of death among wild giant pandas from 2001-2005 highlighting its significance for free ranging animals (Zhang et al. 2008).

We have also recorded parasites resembling Angiostrongylus, a parasite that does not affect the gastrointestinal tract and has been recorded in Red Pandas from various places (Montali et al. 1984; Grondahl et al 2005). Angiostrongylus infects the respiratory tract of dogs, foxes, wolves, coyotes, badgers and wild cats, and its larvae pass the GI tract on their exit passage from the host. Snails, slugs, or frogs are the intermediate hosts of this parasite (Bolt et al. 1992; Patterson-Kane et al. 2009). This nematode has been found to be an underlying cause of respiratory problems including sometimes fatal dyspnoea and pneumonia in Red Pandas (Grondahl et al. 2005; Patterson-Kane et al 2009; Bertelsen et al. 2010). Because the morphology of this parasite is similar to the first stage larvae of Crenosoma, further studies including molecular assays and polymerase chain reaction (PCR) are required to distinguish them. The prevalence of this parasite in our study was low compared to other studies, possibly because we did not use the Baermann method or a PCR assay (Bertelsen et al. 2010).

Finally, Trichuris spp. were commonly found in the samples. This parasite has been reported from humans, pets, livestock and wild animals such as foxes and dogs. Various species of this genus can induce acute or chronic inflammation of the mucosa of the caecum and colon, bloody colitis, lethargy, weight loss, anaemia, mucous, watery or haemorrhagic diarrhoea and dehydration that may lead to the death of animal (Traversa 2011). Notably, further studies should be conducted to assess its pathology in Red Pandas.

Our study has a few limitations. For example, we have not identified parasites to species level. Secondly, the number of faecal samples was low. Despite these shortcomings, we have maintained some qualitative rules of faecal analysis, for example we conducted repeated examinations, acid-fast staining and sporulation assays. On the basis of this exploratory study, we suggest that the health of the Red Panda in this Himalayan region might be compromised due to a high parasitic burden. The prevalence of coccidian parasites resembling Cryptosporidium and Cyclospora (Ghimire \& Sherchan 2006) could be an indicator for a compromised immune 
system. Nevertheless, further analyses are required to identify the preliminary diagnosed parasites and assess their pathological consequences in Red Pandas.

\section{CONCLUSIONS}

The current study highlights the precarious state of health of Red Pandas in the wild. A parasite burden of this degree could seriously compromise the fitness of the animals. Therefore, a deworming program should be considered in this area. Avoiding cattle grazing near the Red Panda habitat would prevent transmission of zoonotic parasites. Contact with snails cannot be fully eliminated but mechanical and chemical methods to minimize the exposure of the pandas to snails should be explored. Further studies including the collection and analysis of large numbers of faecal samples samples and their histological and molecular analysis will bring information regarding the taxonomic position of the parasites and the epidemiological links between the feeding habits of Red Pandas and these parasites. Our study hopefully can contribute to a new level of disease management in Red Pandas in their wild habitat.

\section{REFERENCES}

Bertelsen, M.F., F. Meyland-Smith, J.L. Willesen, R. Jefferies, E.R. Morgan \& J. Monrad (2010). Diversity and prevalence of metastrongyloid nematodes infecting the Red Panda (Ailurus fulgens) in European zoos. Veterinary Parasitology 172 (3-4): 299304; http://dx.doi.org/10.1016/j.vetpar.2010.04.043

Bolt, G., J. Monrad, P. Henriksen, H.H. Dietz, J. Koch, E. Bindseil \& A.L. Jensen (1992). The Fox (Vulpes vulpes) as a reservoir for canine angiostrongylosis in Denmark. Field survey and experimental infections. Acta Veterinaria Scandinavica 33: 357-362.

Choudhury, A. (2001). An overview of the status and conservation of the Red Panda Ailurus fulgens in India, with reference to its global status. Oryx 35(3): 250-259; http://dx.doi.org/10.1046/j.13653008.2001.00181.x

Eberhard, M.L., N.J. Pieniazek \& M.J. Arrowood (1997). Laboratory diagnosis of Cyclospora infections. Archives of Pathology and Laboratory Medicine 121: 792-797.

Ghimire, T.R. \& J.B. Sherchan (2006). Human infection of Cyclospora cayetanensis: A review on its medico-biological and epidemiological pattern in global scenario. Journal of Nepal Health Research Council 4 (2): $25-40$.

Gonzalez-Suarez, M. \& E. Revilla (2014). Generalized drivers in the mammalian endangerment process. PLoS ONE 9(2): e90292; http:// dx.doi.org/10.1371/journal.pone.0090292

Grondahl, C., J. Monrad, H.H. Dietz, H.E. Jensen, M.V. Johansen \& C. Kapel (2005). Angiostrongylosis in Red Panda (Ailurus fulgens fulgens). International Symposium. Paper presented at Internationalen Symposiums über die Erkrankungen der Zoo- und Wildtiere, Erkrankungen der Zootiere, Prag, Czech Republic.
Johnson, K., G. Schaller \& J. Hu (1988). Comparative behavior of Red and Giant Pandas in the Wolong Reserve, China. Journal of Mammology 69: 13.

Kramer, M.H., G.J. Greer, J.F. Quinonez, N.R. Padilla, B. Hernandez, B.A. Arana, R. Lorenzana, P. Morera, A.W. Hightower, M.L. Eberhard \& B.L. Herwaldt (1998). First reported outbreak of abdominal angiostrongyliasis. Clinical Infectious Diseases 26: 365-372.

Montali, R.J., M. Roberts, R.A. Freeman \& M. Bush (1984). Pathology survey of the Red Panda (Ailurus fulgens), pp. 128-140. In: Ryder, O.A. \& M.L. Byrd (eds.). One Medicine. Springer, Berlin Heidelberg.

Nowak, R.M. (1999). Walker's Mammals of The World. Johns Hopkins University Press.

Patterson-Kane, J.C., L.M. Gibbons, R. Jefferies, E.R. Morgan, N. Wenzlow \& S.P. Redrobe (2009). Pneumonia from Angiostrongylus vasorum infection in a Red Panda (Ailurus fulgens fulgens). Journal of Veterinary Diagnostic Investigation 21: 270-273.

Pedersen, A.B., K.E. Jones, C.L. Nunn \& S. Altizer (2007). Infectious diseases and extinction risk in wild mammals. Conservation Biology 21: 1269-1279.

Pradhan, S., G.K. Saha \& J.A. Khan (2001). Ecology of the Red Panda Ailurus fulgens in the Singhalila National Park, Darjeeling, India. Biological Conservation 98(1): 11-18.

Smith, K.F., D.F. Sax \& K.D Lafferty (2006). Evidence for the role of infectious disease in species extinction and endangerment. Conservation Biology 20: 1349-1357; http://dx.doi.org/10.1111/ j.1523-1739.2006.00524.x

Steffens, E. (2004). Red Pandas and Conservation, Political Ecology, Tenure, Livestock, and Hunting in High Altitude Forests of Nepal. University of Wisconsin-Madison, 278pp.

Sutherland, W.J. (1996). Ecological Census Techniques: A Handbook. Cambridge University Press, 336pp.

Traversa, D. (2011). Are we paying too much attention to cardiopulmonary nematodes and neglecting old-fashioned worms like Trichuris vulpis? Parasites and Vectors 4: 32; http://dx.doi. org/10.1186/1756-3305-4-32

Wang, X., A. Choudhury, P. Yonzon, C. Wozencraft \& T. Zaw (2008). Ailurus fulgens. The IUCN Red List of Threatened Species. Version 2015.1. <www.iucnredlist.org>. Downloaded on 18 June 2015.

Wei, F., Z. Feng, Z. Wang \& J. Hu (1999). Current distribution, status and conservation of wild Red Pandas Ailurus fulgens in China. Biological Conservation 89: 285-291.

Williams, B. (2006). Red Panda in eastern Nepal: how does it fit into ecoregional conservation of the eastern Himalaya? Conservation Biology in Asia, Society for Conservation Biology and Resources Himalaya, Kathmandu, Nepal.

Yonzon, P.B. (1989). Ecology and Conservation of the Red Panda in the Nepal-Himalayas. PhD Thesis. University of Maine.

Yonzon, P.B. \& M.L. Hunter (1991). Cheese, tourists, and Red Pandas in the Nepal Himalaya. Conservation Biology 5: 196-202.

Zanini, G.M. \& C. Graeff-Teixeira (1995). Abdominal angiostrongyliasis: its prevention by the destruction of infecting larvae in food treated with salt, vinegar or sodium hypochlorite. The Journal of the Brazilian Society of Tropical Medicine 28: 389-392.

Zhang, J.S., P. Daszak, H.L. Huang, G.Y. Yang, A.M. Kilpatrick \& S. Zhang (2008). Parasite threat to panda conservation. Ecohealth 5: 6-9.

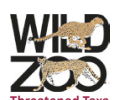

